\title{
Acquisition, tracking, pointing, and line-of-sight control laboratory experiments for a space-based bifocal relay mirror
}

\author{
M. G. Spencer*, B. N. Agrawal, M. Romano \\ Spacecraft Research and Design Center \\ Naval Postgraduate School \\ Monterey, CA 93943
}

\author{
R. L. Brunson**, J. D. Dillow, D. H. Nelson, J. J. Connors, Boeing SVS \\ Albuquerque, NM \\ S. R. Restaino*** \\ Directed Energy Directorate \\ Air Force Research Laboratory \\ Albuquerque, NM
}

\begin{abstract}
Space based bifocal relay mirrors are potentially an enabling/enhancing piece of any architecture making use of longrange laser propagation. Inherent in the bifocal concept is dual line of sight control. This is especially challenging in this space-based application due to spacecraft attitude control issues. This paper presents a summary of the research into acquisition, tracking, pointing (ATP) and control technologies relevant to a bifocal relay mirror system as well as the development of a laboratory experimental test bed to integrate the advanced optics systems onto a Three-axis spacecraft simulator. The relay geometry includes a cooperative source and either a cooperative or non-cooperative target depending on the application. The described test bed is a joint effort with the Air Force Research Laboratory (optics) and the Naval Postgraduate School (spacecraft simulator).
\end{abstract}

\subsection{INTRODUCTION}

A Bifocal Relay Mirror spacecraft is composed of two optically coupled telescopes used to redirect the laser light from ground-based, aircraft based or spacecraft based lasers to distant points on the earth or in space for a variety of force enhancement missions. Students in the Spacecraft Design course at the Naval Postgraduate School did a preliminary design of such a spacecraft in the summer 2000 with AFRL sponsorship. The spacecraft consists of set of single axis gimbaled receive and transmit telescopes with $1.64 \mathrm{~m}$ diameter primary mirrors. The transmit telescope has a majority of the spacecraft bus subsystems including the attitude control actuators and sensor, reaction wheels and star trackers. The spacecraft mass is $3300 \mathrm{~kg}$ at launch and the planned operational altitude is $715 \mathrm{~km}$. The mission requirements are for a 3-m spot beam on the ground, jitter less than 144 nanoradians, and 250 seconds mean dwell duration per pass. The spacecraft design is shown in Figure 1. The preliminary design effort identified the need to develop several new technologies for this mission in the areas of acquisition, tracking, and pointing and beam control optics.

The objective of this research effort is to evaluate different paths in the development of acquisition, tracking and pointing (ATP) and beam control optics technologies for the Bifocal Relay Mirror Spacecraft. The challenge in ATP results from the spacecraft having two space telescopes with interconnected optics and each of theme having different

\footnotetext{
* mgspence@nps.navy.mil

**dick.brunson@svsinc.com

*** sergio.restaino@kirtland.af.mil
} 
targets, with one uncooperative target, and with precision pointing and jitter requirements. The emphasis of this effort was to perform preliminary development work on the different aspects of the control systems and to design a test bed for future development in ATP technologies. A team from NPS, AFRL and several support contractors was assembled with strengths in spacecraft attitude control, vibration isolation and adaptive optics. This paper presents a summary of the research and analysis of ATP technologies, the integrated control simulations and analysis as well as the development of a test bed for a bifocal relay mirror spacecraft.

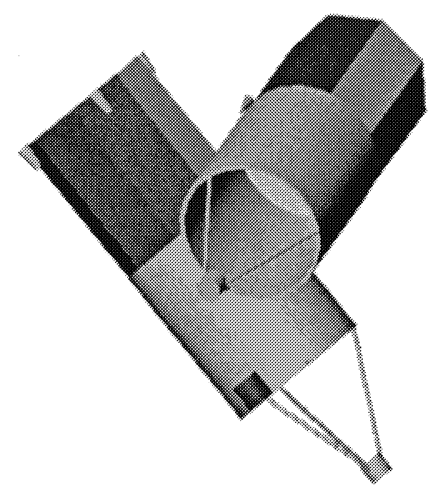

Figure 1. Bifocal Relay Mirror Spacecraft from NPS student design project

\subsection{BEAM AND POINTING CONTROL}

The performance of laser optic systems depends on how well jitter can be reduced or eliminated. Jitter performance is given in terms of the strehl ratio of the optics system in the farfield. Strehl ratio is defined as the ratio of the peak intensity of the transmitted beam in the farfield divided by the peak intensity of an ideal transmitted beam with no degradation. The current design of the bifocal relay mirror (1.64 m aperture) yields a jitter requirement of 140 nanoradians. Attaining these levels of jitter control and vibration reduction requires the use of inertial platforms to

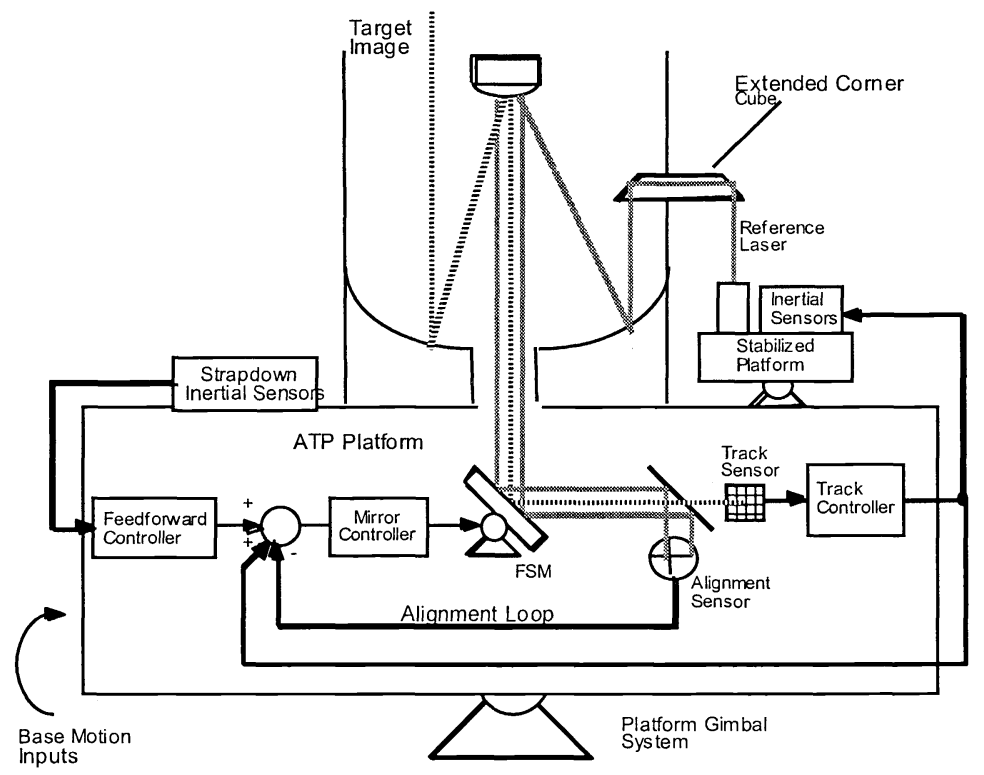

Figure 2. Inertial Reference Platform concept for laser system stabilization 
provide the precision references for the optical system relative to inertial space. Technologies in ATP and beam control optics used or under development on other programs such as the Hubble Space Telescope, Space-based Laser (SBL), Airborne Laser (ABL), and Ground-based Laser (GBL) were evaluated in terms of applicability to the Bifocal Relay Mirror spacecraft. An example of the inertial reference platform is shown in Figure 2.

Precision pointing and low jitter technology has evolved with the development of high energy lasers that date back to programs in the 1970's and 1980's. An example of early programs includes the Relay Mirror Experiment (Laser beam from ground to space to ground) [Ref 1]. Inertial reference platforms and high bandwidth steering mirrors have continued to improve since these early research programs.

The ability of an optical system to produce high fidelity and high-resolution images or to focus the light, in the case of beam projection, is the most important aspect of the optical system itself. At optical wavelengths large telescopes, used for astronomical or surveillance applications, are rarely limited by the intrinsic diffraction effects, but by the presence of aberrations that degrade the impinging wavefront. In order to overcome this dramatic limitation several techniques have been developed. These techniques can be grouped into two broad groups: the pos-facto group and the real time one. To the first category belong all the various deconvolution algorithms that have been developed. However, in order to increase the capabilities of an optical system, both from the imaging or beam projection point of view, it is imperative to use real time techniques. All these techniques are usually referred to as beam-control or adaptive optics (AO) techniques. Until recently beam control was thought mostly in terms of a closed loop system, based on a wavefront sensor and a deformable mirror (DM).

A large body of literature can be found on the use of conventional adaptive optics. However, especially for spaceborne missions the need of using non-mechanical means is becoming increasingly evident. In this framework the AFRL/DEBS group has been working on developing non-mechanical means for both beam control and beam steering. Two main areas of research have been tested and evaluated: Liquid Crystals (LC) and Micro-Electro-Mechanical (MEM) devices.

The idea of using Liquid Crystals for adaptive optics components is becoming more widely accepted. Especially considering that a body of experimental and theoretical evidence on the use of such devices for wavefront correction has been gathering in the recent years. For simplicity sake we will divide the liquid crystals of interest for electro-optical applications in two broad categories: phase modulators and intensity modulators. For applications in $\mathrm{AO}$ we will focus on the first category. Within this category we can distinguish two types of LC materials the nematic and smectic (ferroelectric) phases. In a general way we can regard the nematic as acting like a linear waveplate with an electrically controllable retardance, the ferroelectric as a linear waveplate with an electrically controllable optical axis. Both type of $\mathrm{LC}$ can be used in an AO system.

\subsection{ANALYTICAL DYNAMIC MODEL}

A Matlab/Simulink based analytical dynamic model of the bifocal relay mirror system was developed. The system is a multi-body dynamics problem consisting of two main bodies; the transmit and receive telescopes. The transmit telescope rotates with respect to the receive telescope about a single axis. Each telescope is assumed to have a fast steering mirror and the transmit telescope has reaction wheels. The bodies are assumed to be rigid bodies. The spacecraft model is shown in Figure 3.

The equations were written in the transmit telescope fixed coordinate frame. The spacecraft is subjected to large angle maneuvers; therefore, a quaternion formulation is used. The spacecraft uses rate gyros in the transmit telescope to determine angular rates and star trackers to determine angular positions. The rate gyros have bias errors and star trackers have measurement gaps. During the measurement gaps for the star trackers, rate gyros are used to determine angular rates and angular position. When the star tracker measurements are taken, using a Kalman filter, the angular positions are corrected and the rate gyro bias are updated. Magnetic torquers are used to desaturate the reaction wheels. The controller uses a feed forward control torque developed by neglecting the effects of fast steering mirrors (small inertia). The wheel control laws are based on quaternion error and angular velocity error feedback. The derivation of the equations of motion and simulation models are presented in Ref 2. 


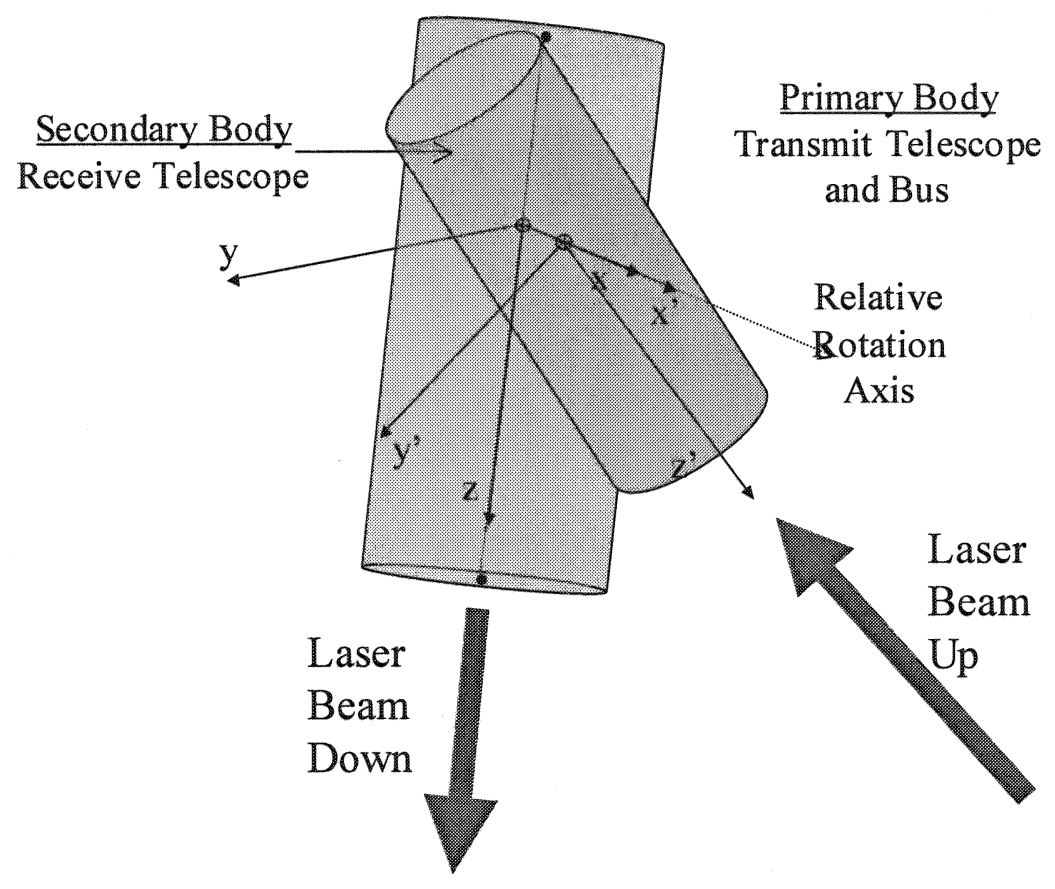

Figure 3. Relay Mirror Spacecraft Model

\subsection{POINTING CONTROL AND ANALYTICAL SIMULATIONS}

In this section, several line-of-sight control options were discussed for doing the integrated satellite and optical control functions for the Bifocal Relay Mirror concept. The assumptions used in the following discussion are as follows.

The transmitter will be nominally pointing to a set of pre planned ground targets during a pass. In more stressing missions, the transmitter will be pointing at moving targets. This telescope is controlled using Reaction Wheels (or CMGs). The spacecraft bus will be attached to the transmitter telescope using a hexapod structure. Options for the hexapod include passive damping or active control in order to isolate the bus disturbances from the optical system. The bus disturbances include the reaction wheels, cryo-cooler for the infrared tracking sensor, and solar cell motion. The receiver will be pointing at a fixed ground based laser location or another Bifocal Relay in orbit. The receiver telescope is controlled using a torque motor to rotate the receiver relative to the transmitter.

The trackers are assumed to be "shared aperture" type trackers, meaning that they will use the full aperture of each telescope to image the "target" in the case of the transmitter aperture and the laser source, or beacon, in the case of the receiver aperture. This will require an aperture sharing element, which provides the appropriate separation of the light of the relay laser from the light used for the tracker.

The receiver tracker is assumed to be a centroid tracker to track a beacon or the relay laser. This tracker will have high SNR and could have a high frame rate. The transmitter tracker is assumed to be a correlation tracker to track a scene in the infrared waveband. This tracker will have medium SNR and a lower frame rate. Details of how to insure the alignment of the two focal planes and how to precisely control the laser point ahead are not discussed in this section. The Receiver and Transmitter may have additional apertures and trackers to assist in source and target acquisition, depending on the cost trades between the inertial sensors and optical sensors. If there is uncertainty in the target position, then a separate aperture acquisition tracker with a wide field of view is likely required for the Transmitter Telescope. 
The control options were integrated into the Matlab/Simulink model of the Bifocal Relay Mirror Spacecraft. Different combinations of control architectures were simulated to evaluate the effectiveness of the integrated control system. The baseline system involved independent control of the fast steering mirrors and spacecraft attitude. The results were compared against those for the integrated control of the transmitter fast steering mirror and spacecraft attitude. With this control approach, the transmitter fast steering mirror position relative to the structure is measured and used to control the spacecraft attitude.

Two different sample cases were studied in the simulations: 1) Target and source in certain position and 2) Target in an uncertain position. The simulations cover a 500 second time period in which the controller must drive the transmit telescope to track the target while moving the receiver telescope to maintain lock with the source laser.

The first control approach involved independent control of fast steering mirrors and spacecraft attitude. Using this approach, the spacecraft attitude is controlled independently of the fast steering mirrors motion. While the spacecraft attitude is controlled by feed-forward and PD commands, sensing the attitude error with the IRU (composed by star trackers and rate gyros), the transmitter and receiver fast steering mirrors are controlled by a PID control, sensing the target and source errors with optical sensors (target and source tracker). The use of a feed-forward command to control the spacecraft is a new point with respect to ref.1, where only the PD control was envisioned for the spacecraft.

For the second control approach, the transmitter fast steering mirror position relative to the structure is measured and used to control the spacecraft attitude. Indeed, the mirror position is proportional to the target pointing error. The angular position around the yaw axes and the angular rate data, for the attitude control, still comes from the IRU sensors. As regards the receiver fast steering mirror is still controlled independently. The block diagram in Figure 4 describes the concept of this control approach. In the simulation with fixed fast steering mirror, we considered directly the output of the target as a feedback signal.

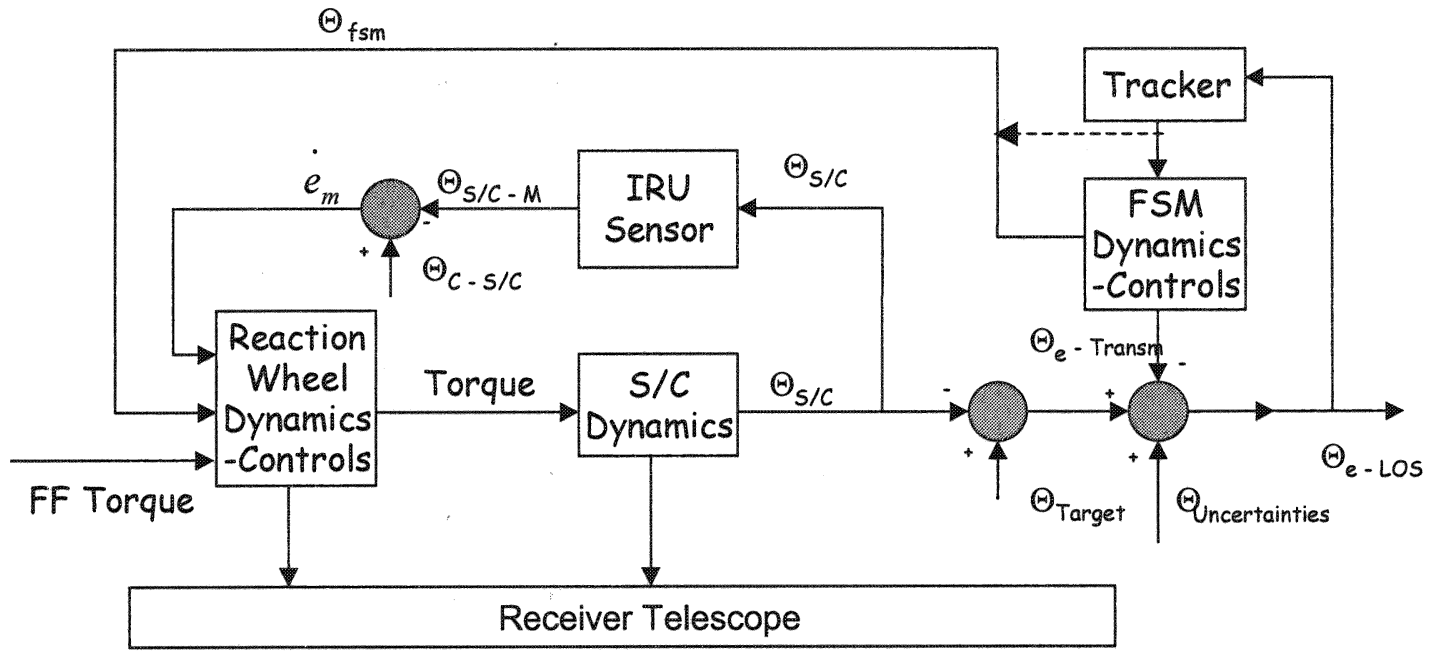

Figure 4: Integrated spacecraft-transmitter fast steering mirror control approach

An example of the simulation results is shown in Figure 5. Based on the results obtained in the simulations, it appears that the best solution to control the Bifocal Relay Mirror system is probably a trade-off between the independent spacecraft-fast steering mirrors control and the integrated spacecraft-transmitter fast steering mirror control. That is a control that uses a fusion of the data from the target and source tracking sensors and the data from the IRU sensors, weighted on the base of the knowledge of the characteristics of the specific sensors. 

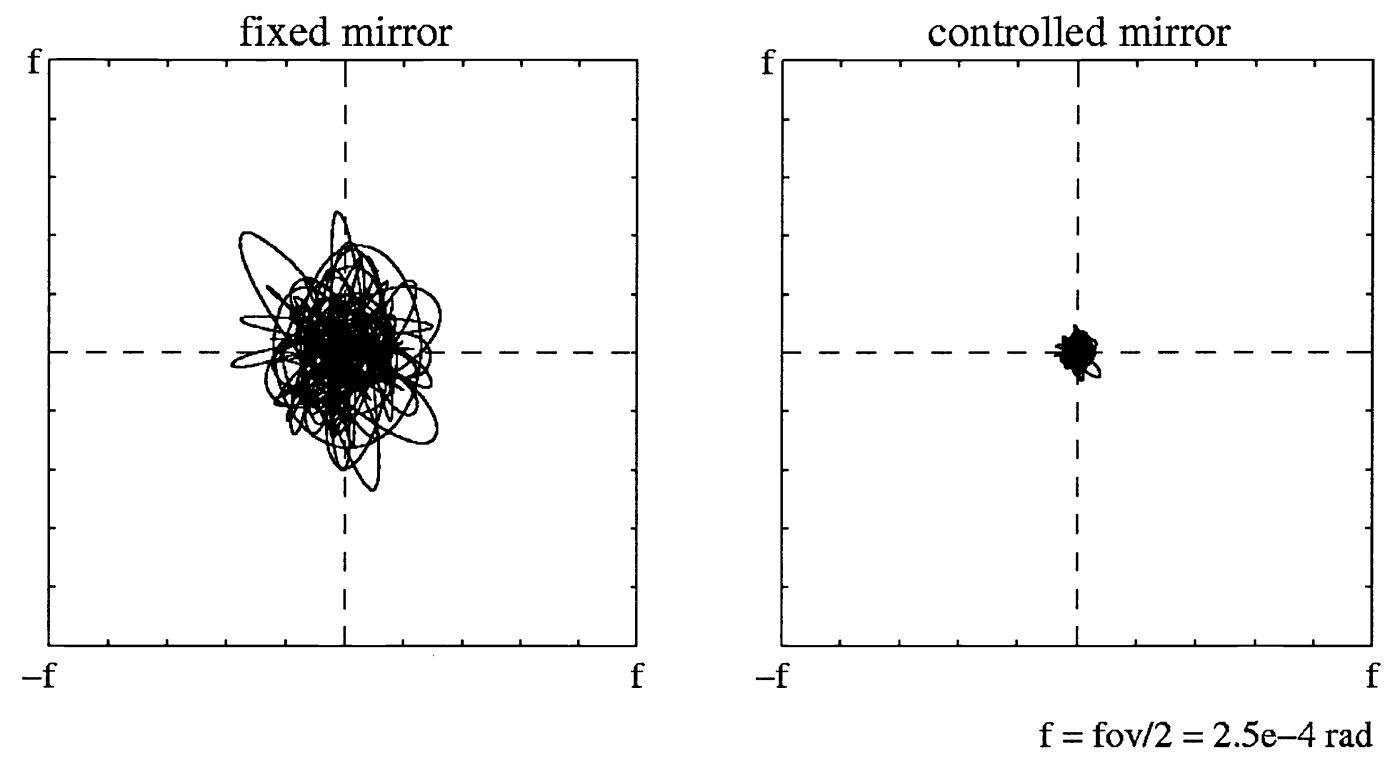

Figure 5. Track of the target on the target sensor. Spacecraft with realistic IRU and Kalman filter. Uncertain knowledge of the target position. Integrated spacecraft - transmitter fast steering mirror control.

\subsection{EXPERIMENTAL TEST-BED}

The objectives of the experiments were to validate the acquisition, tracking and pointing control of a laser beam using steering mirrors integrated onto a moving platform, the NPS Three-Axis Spacecraft Simulator (TASS). The TASS is a platform with reaction wheels, thrusters, rate gyros, sun sensor and control computer that can simulate the attitude dynamics of a spacecraft in orbit by floating on a spherical air bearing. AFRL provided fast steering mirrors, optics, sensors, lasers and the beam control system while NPS provided the TASS as a movable spacecraft platform for the steering mirror and optics. The experiments were divided into phases that involved increasing levels of complexity and fidelity. The experiments were designed as a basic technology demonstration for integrating and controlling a moving relay mirror system and only a single relay mirror system was used.

The experiments were divided into three phases:

Part A: Demonstrate beam jitter control with fast steering mirror on the TASS

Part B: Track a moving target with cameras and fast steering mirror on TASS

Part C: Demonstrate jitter control and target tracking with TASS platform floating and holding a fixed attitude.

In each experiment, the laser source was fixed and placed 2-3 meters from the TASS. During Parts A and B the TASS was held fixed and did not float. Part $\mathrm{C}$ experiments were conducted with the TASS floating and the laser source fixed off the platform. Target tracking and controlled laser operations were conducted with the TASS platform independently maintaining a fixed attitude. The optical system and platform control system were not integrated.

Elements of the Part A experiments were conducted at AFRL with the jitter control system on a typical optical bench. The fast steering mirror and jitter control system were shipped to NPS and integrated onto the TASS. The tests successfully minimized the beam jitter caused by vibrating the source laser. Part B and C experiments involved the tracking of a green laser designator in a target screen with a camera system. A separate, off-board control computer processed the image and commanded the fast steering mirror to direct the red source laser to follow the target. During Part B tests, the TASS was fixed. Figure 6 shows the schematic of the test setup. 


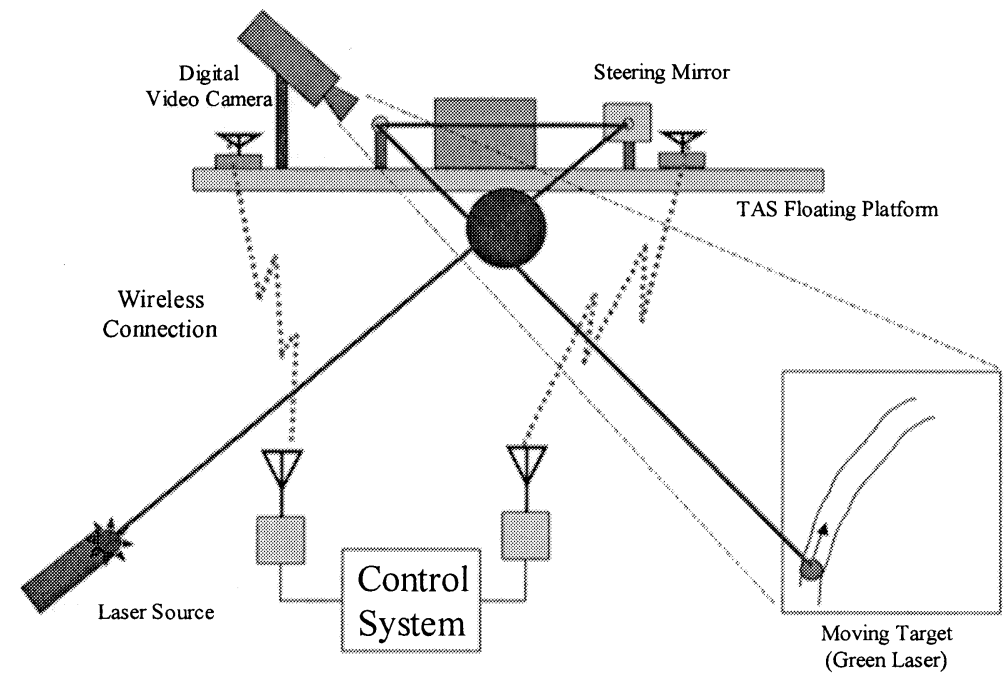

Figure 6. Part B/C Target tracking test configuration

The Three-Axis Satellite Simulator consists of several subsystems that act together to simulate satellite functions and attitude dynamics. The base structure is an octagonal aluminum plate, .375" thick. A ten-inch diameter spherical air bearing is rigidly attached to the underside in the center of the plate. This air bearing sits in an air-bearing cup, which provides a smooth surface for the bearing to rest in when air is not applied to the cup. When air is applied to the cup, it raises the table $3 / 8$ " to a free-floating position.

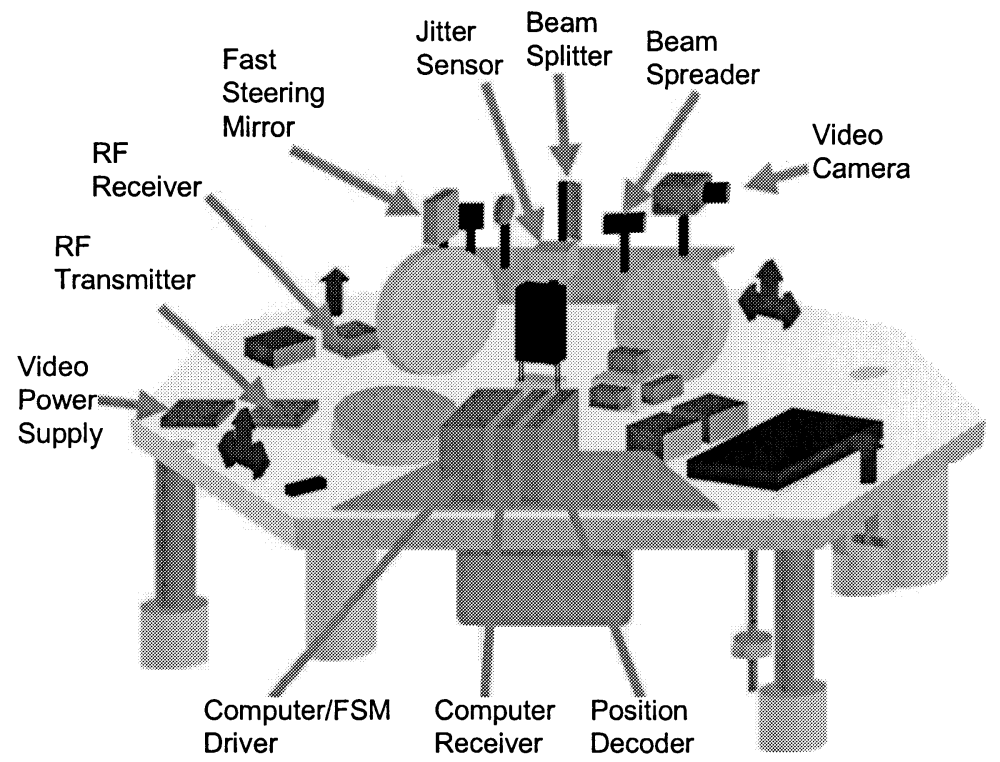

Figure 7. Relay Mirror Payload Layout on TASS

Subsystems on the table include three orthogonally mounted reaction wheels, three orthogonally mounted rate gyros, a three-axis sun sensor, a three-axis magnetometer, a laptop computer, three lead-acid batteries, and a thruster system of two nitrogen propellant tanks and four thruster blocks providing three-axis control. Figure 7 outlines the locations of these components and Figure 8 is a top view picture of the integrated Optics/TASS system. 


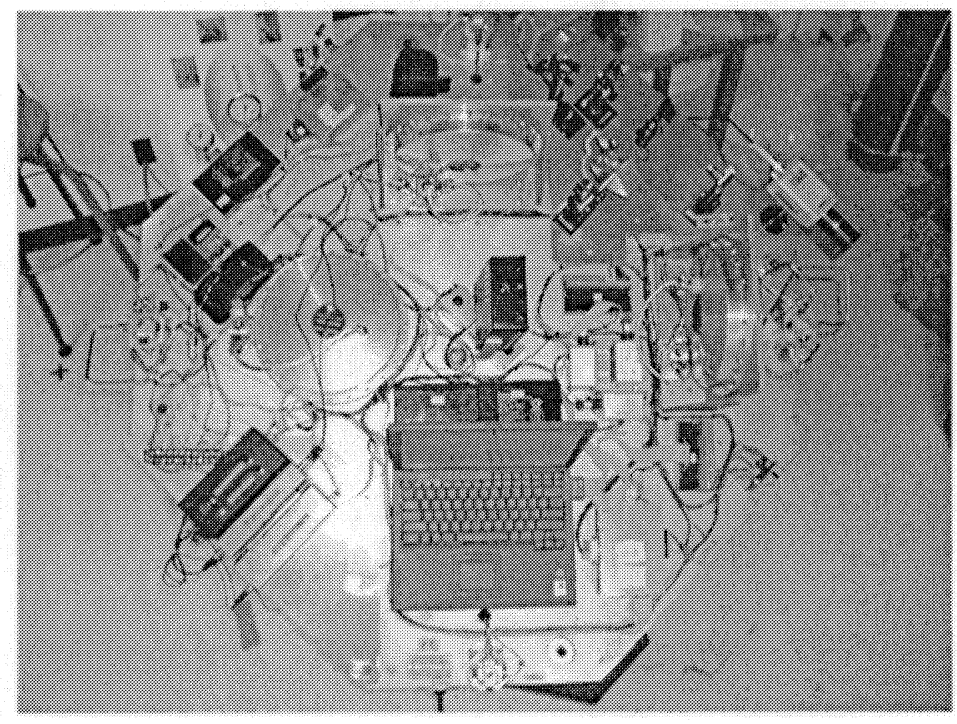

Figure 8. TASS Top View

The remaining experimental efforts include the integration of the optics/camera power and control system to the TASS platform. The TASS attitude-hold control program was improved to maintain a stable platform during the active target tracking tests. One fast steering mirror was used to control both jitter and target tracking. The one-inch diameter mirror and source laser placement resulted in an attitude-hold requirement of 0.1 degree to keep the source laser on the steering mirror.

The TASS initially had a two-axis sun sensor mounted to the left-hand side of the laptop computer on the platform. During initial testing, it was determined insufficient data existed to stabilize the TASS using sun sensor and rate gyro data alone. The sun sensor was removed and modified to incorporate a third axis of information. The sun sensor was remounted in the center of the table facing the ceiling. A 12 foot blackout canopy was built 5 feet above the table, and two lights roughly one foot apart were placed in the center of this canopy to simulate the sun and a single star. The sun sensor used one of these lights to provide roll and pitch position information, and the position of the second light relative to the first light to provide yaw information.

The optical train consists of a primary mirror, the Fast Steering Mirror (FSM), a lens, a variable beam splitter, a jitter sensor, and a beam spreader. A video camera is mounted adjacent to the optical train to provide feedback for beam targeting and steering. A diagram of the optical train is contained in Figure 9. The beam spreader is an inverted microscope lens, amplifying small motions of the FSM into larger motions on the target (A map of the world, hung on a wall ten feet away).

The red bench-top laser is aimed at the primary mirror, which directs the laser at the FSM and then through a beam spreader to the target on a wall in the lab. Onboard the platform, the laser beam passes through a splitter, and highfrequency jitter is sensed and removed with a closed loop controller to the FSM. The video camera sends an image of the target to the control computer (located off the floating platform), which processes the image and sends fast steering mirror commands back to the floating platform to drive the red laser beam towards the green laser target. 


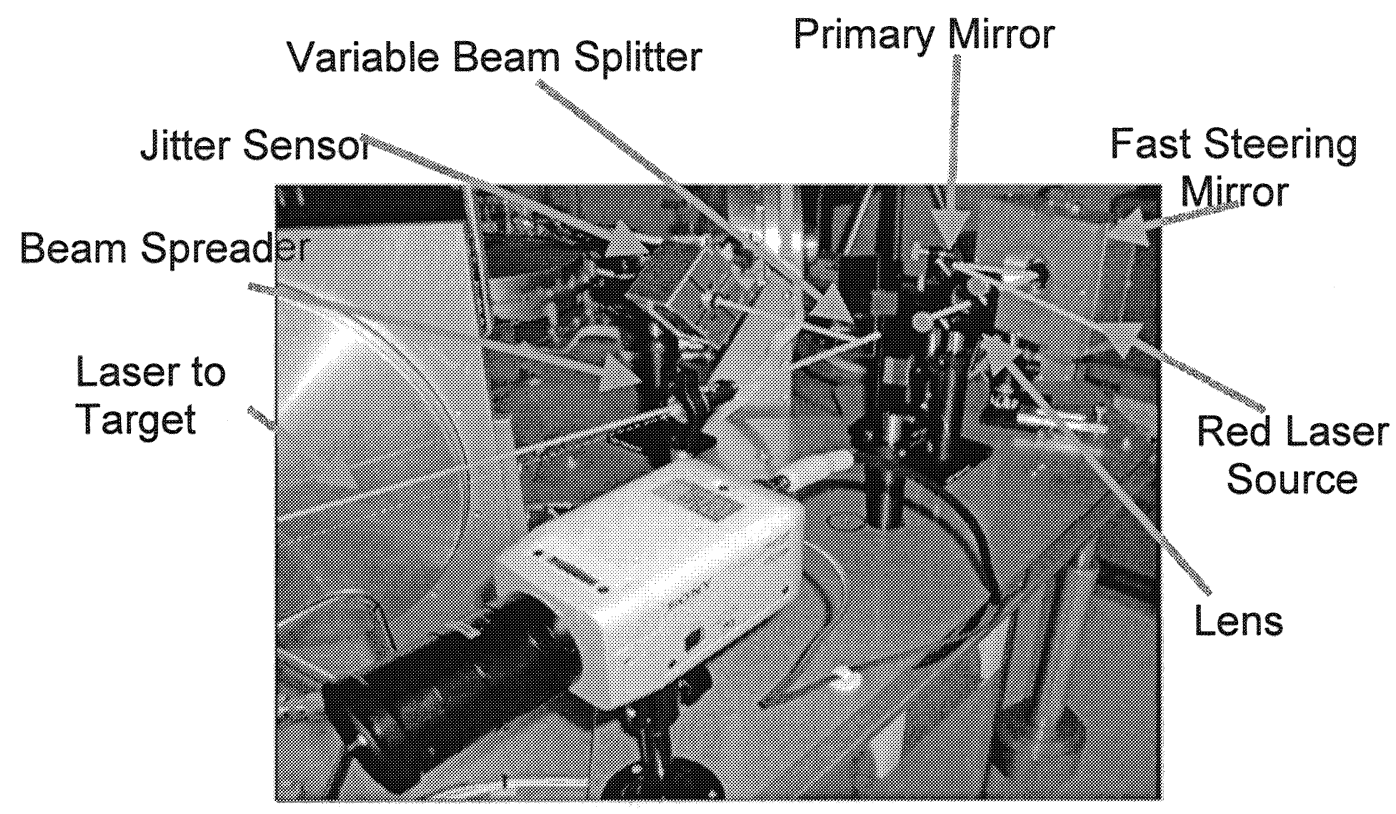

Figure 9. Optical Train Diagram

Successful completion of Part $\mathrm{C}$ experiments were demonstrated with the target tracking of the green designator with the red source laser while the TASS was free floating. These experiments successfully demonstrated the closed loop control of the source laser to track the green target spot. The target tracking control system was developed at AFRL and control panel interface is shown in Figure 10 [Ref. 3].

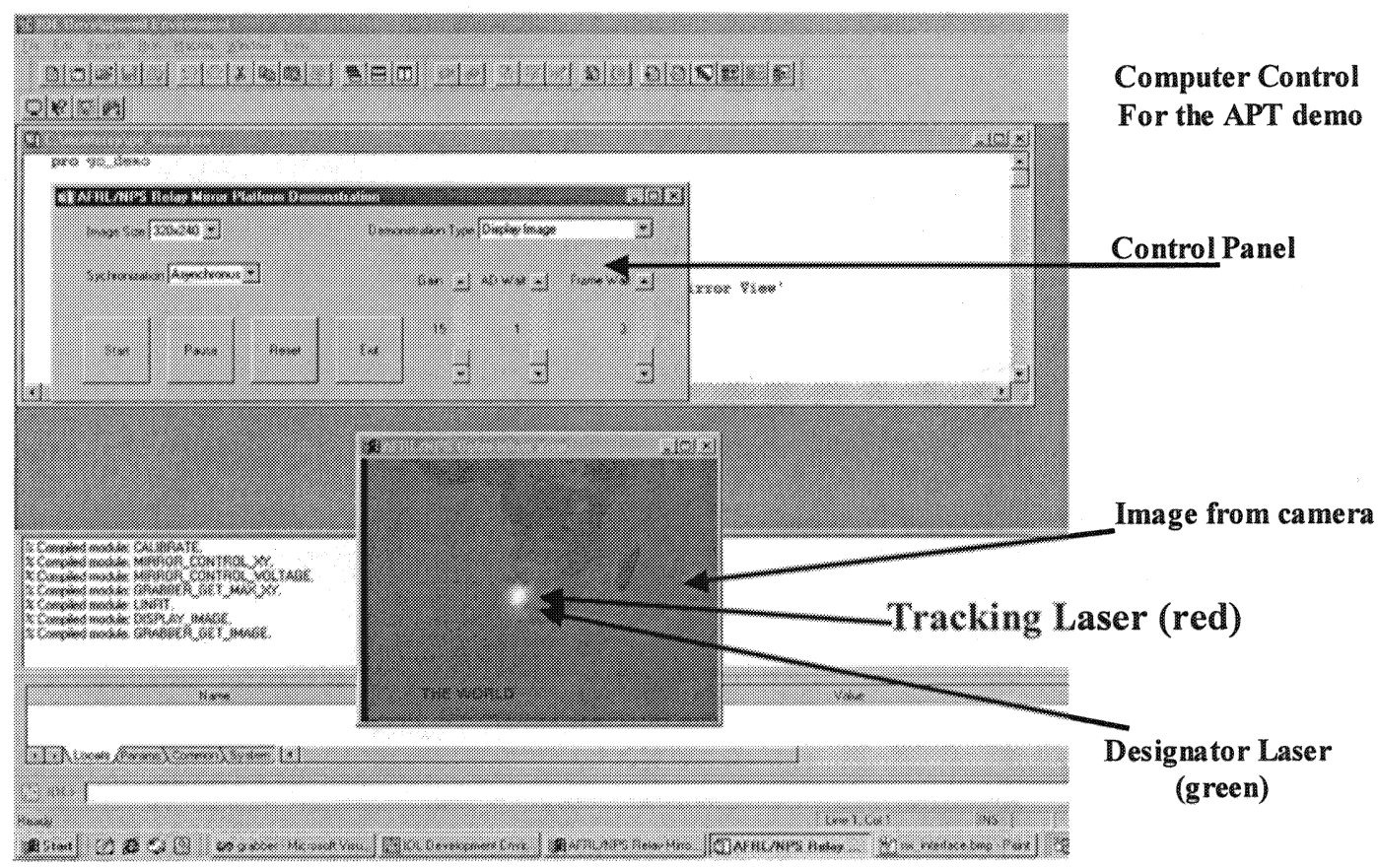

Figure 10. Target tracking computer control panel with inlet pane of tracking image 


\subsection{Future Technology Development Efforts}

Considerable experience and insight was gained from the previous efforts. A test bed is critical for integrating ATP technologies and testing system performance. The current test bed at NPS integrates spacecraft, fast steering mirror, and target tracking technologies. However, it has several limitations. The attitude sensor, sun sensor, has pointing accuracy of only $0.1 \mathrm{deg}$. The controls of spacecraft, fast steering mirror, and target tracking controls are not integrated and only one fast steering mirror is used. Target tracking is limited by using a laser designator on the target. A future test bed to demonstrate the relay mirror scissor configuration will require a new modified version of a platform on a spherical air bearing. The current ideas for a follow-on test-bed are shown in Figure 11. The new test bed would make use of the some of the current simulator hardware while integrating a dedicated telescope and fast steering mirror system.

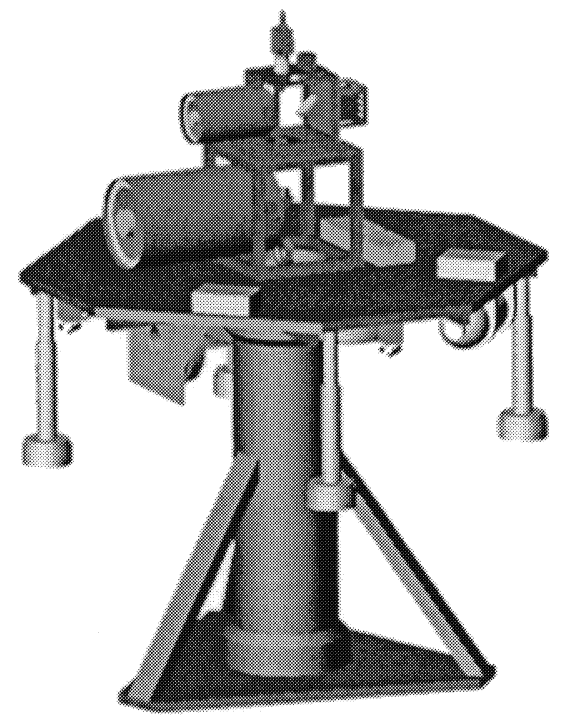

Figure 11. Bifocal Relay Mirror Test-bed Design Concept

The objectives of the current effort were to evaluate different paths in the development of fine acquisition, tracking, and pointing (ATP) and beam control optics technologies for the Bifocal Relay Mirror spacecraft, do preliminary development work, and design a test bed for the development of these technologies. All the objectives of the current effort have been met. Significant future effort is required to develop these technologies in several areas and in several phases. These technologies can be directly used for other spacecraft with optical payloads such as imaging spacecraft and spacecraft for laser communications. The recommended areas for future technology development are as follows.

The analytical simulation has to be improved by using more advanced control architectures such as Inertial Reference Units (IRU) and Auto Alignment (AA) loops. As an example the control architectures include tracker errors controlling telescope pointing, AA loop controlling fast steering mirror (FSM); AA loop controlling FSM, tracker errors controlling IRU on flexure, IRU position controlling telescope pointing.

The experimental verification can be achieved in two phases. In Phase I, the current test bed, representing a single telescope, will be upgraded by using more accurate spacecraft sensors. The pointing accuracy of the current sun sensor is only 0.1 degree and rate sensors are noisy. Currently the controls of spacecraft, FSM and tracking are independent. These control need to be integrated using new onboard sensors to test different control architectures. In Phase II, the Bifocal Relay Mirror test bed as shown above will be developed. This will represent both telescopes and they will be optically coupled. Tests will be performed to validate the interaction of the ATP systems of both telescopes.

Jitter is very critical to meet the performance of the optical payload. Both active and passive vibration isolation techniques will be evaluated for application to this spacecraft. Location of the vibration isolation at different locations, such as at the disturbance source, interface between spacecraft bus and payload, and vibration suppression on the 
payload will be evaluated. Improved techniques for integrating vibration isolation and fine steering of FSM will be developed. The Spacecraft Research and Design Center at the Naval Postgraduate is very active in this research area.

\subsection{References}

1) Roberts, S. S., Relay Mirror Experiment and Wideband Angular Vibration Experiment Program Summary, AFRL/DE, Kirtland AFB, NM, Issued: May 1992.

2) Agrawal, B.N., Senenko, C., “Attitude Dynamics and Control of Bifocal Relay Mirror Spacecraft”, Proceedings of the AAS/AIAA Astrodynamics Specialists Conference, Quebec City, Quebec, Aug 2001.

3) Chernesky, V. S., Development and Control of a Three-Axis Satellite Simulator for the Bifocal Relay Mirror Initiative, Masters Degree Thesis, Naval Postgraduate School, Monterey, CA, Dec, 2001. 